\section{Guidelines produced for the use of transgenic animals in research}

\section{London}

WITH transgenic animals finding increasing favour in both biomedical research and biotechnology, the UK Advisory Committee on Genetic Manipulation (ACGM) has produced detailed guidelines on their use. The guidelines, which the committee hopes will set an example for other countries, are particularly concerned with the consequences of the deliberate or accidental release of transgenic animals into the

\section{HIV a problem for Indian blood banks}

\section{New Delhi}

INDIA is suffering from a wave of public alarm caused by news that human immunodeficiency virus (HIV) is increasingly being transmitted by use of donated blood. This development conflicts with the earlier belief that AIDS is being spread chiefly by prostitutes and foreigners.

Officials say that the government can no longer postpone compulsory screening of all blood banks, a suggestion that only six months ago was dismissed as unnecessary as well as impracticable.

No transfusion-related HIV infection had been detected in India until July 1987, which is one reason the government was content with only minimal restrictions on imported blood products. But since then, the proportion of seropositive cases ascribed to transfusion has been increasing rapidly.

Last month alone, the All-India Institute of Medical Sciences in New Delhi discovered antibodies to HIV in three haemophiliac patients, and doctors in another hospital said that 10 of their 6,136 voluntary blood donors tested positive for AIDS. Of the 620 confirmed cases of HIV infection in India, about 60 have received blood transfusions in Indian hospitals.

According to the Indian Council of Medical Research (ICMR), which operates some 40 AIDS surveillance centres, blood transfusion has become an important mode of HIV transmission. An estimated 1.5 million units of blood are used each year in India, but ICMR's centres can perform only 30,000 tests, using kits imported from Britain. Complete blood screening would require a 100 -fold increase in the number of kits. Although ICMR feels that all donated blood must be screened, the job "cannot be done overnight".

As a first step, all donors in Bombay and Madras are now being screened, and experience from these cities will be used to start screening in other cities in about a year, by which time India hopes to be producing its own test kit. K.S. Jayaraman wild, and the hazards of using viral, and especially retroviral, vectors for transgenes - that is, the use of genetically modified viruses to introduce new genes into animals.

ACGM, which will need advance notification of any plans to use viral vectors or to release transgenic animals, warns that it will be tough on both. For animals that are to be released, there will be a ban on the use of any viral vector that contains even a partially functional oncogene and on any form of vector that has even a remote potential to replicate. Plans to use equivalent vectors to produce contained transgenic animals will be considered individually.

Among the considerations will be the precautions that have been planned to ensure containment. For large animals, ACGM takes the view that a well-fenced field is sufficient. Extra care will be needed for fish and other aquatic vertebrates because of the relative ease with which they or their gametes can escape. Even greater precautions are necessary for work with invertebrates that "crawl, jump or fly".

Some of the guidelines on genetic manipulation anticipate changes in the official Health and Safety Regulations, which were drawn up in 1978. Revised regulations suggested by the Health and Safety Commission in October 1987 have since been modified, and now await approval by the Secretary of State and parliament. Their main intent is to establish a legally backed compulsion both to notify ACGM of the production and use of transgenic animals and to set up and operate local risk assessment committees at any establishment that carries out genetic manipulation.

ACGM's guidelines also cover aspects of work with transgenic animals that fall outside its own purview. The welfare of such animals is "dealt with adequately by existing legislation", the guidelines say, pointing out that breeding from transgenic animals is regulated as though an animal experiment until it can be demonstrated that the progeny are not likely to suffer any adverse effects.

The guidelines advise that the consumption of transgenic animals or their products by humans or animals would be subject to consideration by the Advisory Committee on Novel Foods and Processes, which may in turn seek advice from ACGM. Professor Mark Williamson, who chaired the working group that drew up the new guidelines, says it is inconceivable that approval would be given to the consumption of animals containing a human transgene.

\section{Italian environmental programme launched}

\section{Turin}

THE Italian government last week launched a $£ 70$ million programme of research into environmentally benign technology. Research priorities must be formulated within two months by a new commission which will report to the ministries for industry and for the environment which are financing the programme.

The announcement was well-timed, coinciding with an international conference in Turin designed to increase public awareness of the problems of the greenhouse effect, the destruction of the ozone layer and acid rain and to stimulate political action to tackle these problems. Among the recommendations of the conference was a proposal to impose a tax on fossil fuels which would finance a world fund to promote alternative energies and energy efficiency. The conference also recommended improved monitoring of climate change and increasing research into an understanding of the problems. The Italian government was heavily criticized for the lack of support for such research. Money is scarce and research uncoordinated, as well as being hindered by a lack of access to meteorological data, which is controlled by the defence ministry. Christine McGourty

\section{Testing of in vitro embryos approved}

\section{Melbourne}

AN Australian parliamentary committee has approved a controversial request by scientists at the Monash Medical Centre in Melbourne to test human embryos obtained for in vitro fertilization (IVF) for birth defects before implanting them in patients.

Tests will be done about two days after fertilization, when the embryo has grown to four cells. One cell will be removed and analysed for genetic defects. The tests will be performed only on embryos that take longer than usual to fertilize. According to Monash Medical Centre's Professor Carl Wood, the Australian pioneer of IVF, about 3 per cent of embryos have a delayed fertilization, but are not neccessarily abnormal. The tests will prevent normal embryos from being discarded. Embryo biopsy does not seem to damage the survival chances of the remaining embryo.

The decision of the committee, chaired by Professor Louis Waller of Monash University, may cause problems for Victoria Health Minister David White, who has written publicly that there will be no experimentation on human embryos older than 22 hours. The scheme may also face legal problems, as it involves the use of fertilized embryos but is not directly related to alleviating infertility. 